\title{
Risk Measurement of China's SME Board Market Based on GARCH-VaR
}

\author{
Wang Mengnan \\ School of Finance \\ Guizhou University of Finance and Economics \\ Guiyang, China \\ 1004932357@qq.com
}

\author{
Dai Liang \\ School of Finance \\ Guizhou University of Finance and Economics \\ Guiyang, China \\ 780526840@qq.com
}

\begin{abstract}
The GARCH model can describe the dynamic characteristics of the stock market yield better, but the conclusion of the analysis is not the same because of the different sample data selected by the research. On the bas is of the research at home and abroad, this paper selects the China's SME board index which is more familiar to investors, selects the closing price data from June 1, 2006 to June 1, 2017, uses three different types of models, such as GARCH, EGARCH, TARCH, and so on. Under the normal distribution, the students' distribution and the GED distribution, the order of return is the order of return. The column data are fitted and the estimated results are compared and analyzed. It is found that China's SME board index has a more obvious ARCH effect, which is characterized by stationarity, non-normality, peak and thick tail. By comparing the VaR values under different GARCH models, it is found that the GARCH model under the assumption of $t$ distribution can better reflect the risk of China's SME board market. Thus, the overall situation and risk characteristics of the income distribution in the SME board market in China are better obtained, so as to provide the appropriate risk measurement model and decision basis for the developing SME board market.
\end{abstract}

Keywords-SME board; GARCH model; VaR; Normal distribution; $T$ distribution

\section{INTRODUCTION}

Small and medium-sized plates, SMEs board, refers to the circulation of the disk about 100 million of the following entrepreneurial plate, is relative to the motherboard market, some enterprises are not up to the requirements of the motherboard market, so can only be listed in the small and medium-sized market. SME market is a kind of transition of GEM. May 2004, with the approval of the State Council, China Securities Regulatory Commission agreed to the Shenzhen Stock Exchange in the main board market set up SME sector. The establishment of SME board is an important measure to construct multi-level capital market. With the continuous improvement of China's capital market, the increasing liquidity and the drastic fluctuation of market risk, the risk management of China's stock market will become the focus of financial supervision departments and investors. And the volatility of the SME board will be more intense than the motherboard, so the risk research of the SME is also of great significance.

Financial time series tend to fluctuate in a period of time, and in another period of time calm, showing a random walk phenomenon. In theory, this can be abstracted as the volatility of future installments is likely to be greater when the current or past periods fluctuate, and vice versa. In other words, contrasting observations seem to gather together, and small contrast observations seem to gather together, a phenomenon known as "volatility aggregation". In order to measure the volatility of financial time series variance, Engle ${ }^{[1]}(1982)$ presents a autoregressive conditional variance model, which can predict variance better and has important application value in the Financial field. However, in the ARCH model, if the q is large, you need to take into account many parameters, which will lose the sample size. Bollerslev ${ }^{[2]}(1986)$ extended the ARCH model and proposed Generalized ARCH, which made the parameter decrease, so that the prediction of the future conditional variance was more accurate. The GARCH model is a regression model that specializes in the volume of financial data. In addition to the common regression model, the GARCH model is further modeled on the error variance. Especially applicable to the analysis and prediction of volatility, such analysis can play a very important role in the decision-making of investors, the significance of many times more than the analysis and prediction of the value itself. Luo Yang ${ }^{[3]}(2013)$ thinks that the GARCH model can better simulate the peak and thick tail characteristics of the fluctuation, and can eliminate the variance of the stock index fluctuation.

The $\mathrm{VaR}^{[4]}$ method was first introduced by some American financial institutions in $1980 \mathrm{~s}$, and the research on domestic financial risk is relatively lagging behind. Laurent and Peters ${ }^{[5]}$ (2002) use different GARCH models to predict the value at risk $(\mathrm{VaR})$ of the financial market. Xu Wei and Huang Yanlong ${ }^{[6]}$ (2008) used four GARCH models to analyze the VaR prediction results of the Shanghai Composite Index in three different distributions, and found that the GED distribution can better reflect the thick tail characteristics of the Shanghai composite index return sequence. The risk values predicted by the VaR method are usually related to the gains and losses at the end of the yield sequence. When calculating VaR, the probability distribution of the time series is assumed. Considering that most of the financial time series have the characteristics of the peak and thick tail, a large number of studies assume that the sequence is subject to the $t$ distribution or the GED distribution of the students. The conclusions of the analysis are inconsistent because of the different sample data selected by the research Institute. Based on the research at home and abroad, the paper selects the relatively familiar SME board index, adopts three kinds of models such as Garch, 
Egarch and Tarch, and then fits the yield sequence data in normal distribution, student $t$ distribution and GED distribution under three different distributions. The estimation results are compared and analyzed, so that the overall situation and risk characteristics of the market income distribution of China's SME are better obtained, and the accuracy of VAR value calculated by each model under different distribution conditions is given, which provides the appropriate risk measurement model and decision basis for the development of the SME market.

\section{THEORETICAL SUMMARY}

\section{A. The concept and calculation of VaR}

VaR (Value at Risk) usually refers to the maximum loss that an asset or portfolio may suffer at a certain level of confidence at a future time. Represented by a formula:

$$
p(p>\operatorname{VaR})=1-c
$$

Wherein, $\mathrm{C}$ is the confidence level, $\mathrm{p}$ is the asset during the holding period of the loss; VaR is in the risk value. The calculation of VaR usually involves the confidence level, the distribution of asset income and the three basic factors of holding period, and the choice of probability distribution is very important when using parameter method to calculate VaR value. The advantage of $\mathrm{VaR}$ is intuitive and understandable, however, the calculation is more complex, usually assuming that the income of the assets obeys the normal distribution, because of the present value of historical data samples, the assumptions of the model and the error of measurement, the model risk exists in any model, which has the following problems, such as postmortem inspection, stress test and extreme value analysis.

\section{B. GARCH model}

ARCH (Autoregressivec conditional heteroscedasticity models) model was first proposed by Engel (ENGLE.R) in 1982, which reflects a special characteristic of stochastic processes: that variance changes with time and is clustered and volatile. Arch model has been widely used in the modeling and research process of financial field.

The ARCH model is usually used to model the stochastic perturbation term of the subject, and the variance of the stochastic perturbation term is set to the linear function of the square of the error term in the previous period, so that the information in the residual is more fully extracted, so that the final model residuals are white noise.

If the $\mathrm{ARCH}$ model is used to describe some time series and the order needs to take a large value, the generalized autoregressive conditional variance model is usually used.

In practical applications, the order $\mathrm{p}$ of the GARCH model is much smaller than the order $\mathrm{p}$ of the arch model, and the GARCH $(1,1)$ model is a widely used model, which has the following form:

$$
\sigma_{t}=\alpha_{0}+\alpha_{1} u_{t-1}^{2}+\theta \sigma_{t-1}
$$

\section{EMPIRICAL ANALYSIS}

\section{A. Selection of Sample Data}

This paper selects the closing price of the SME composite Index from June 1, 2006 to June 1, 2017, which is a total of 2,677 data as a sample of this empirical analysis. As an investment portfolio of a certain number of stocks, the yield of stock index can be regarded as an important index to measure the volatility of stock market. The value of logarithmic function is the whole real number, which is suitable for financial modeling. This paper chooses logarithmic rate of return to measure the fluctuation risk of China's SME market:

$$
r=\ln p_{t}-\ln p_{t-1}
$$

\section{B. Analysis of the basic characteristics of data}

\section{1) Descriptive statistics}

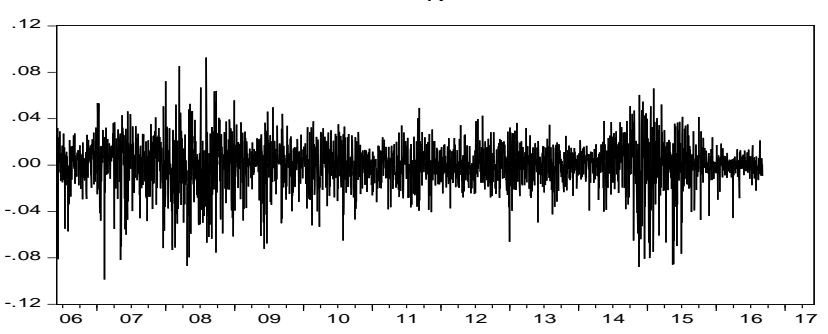

Fig. 1. Logarithmic return time series Chart of the SME Board index

In Fig. 1, it can be found that the small and Medium-sized board index is stable to the return sequence, the fluctuation has cluster phenomenon, the fluctuation is larger in the two time period of 2007 2008 and 2014 2015, but in other time period, the sequence may exist conditional variance.

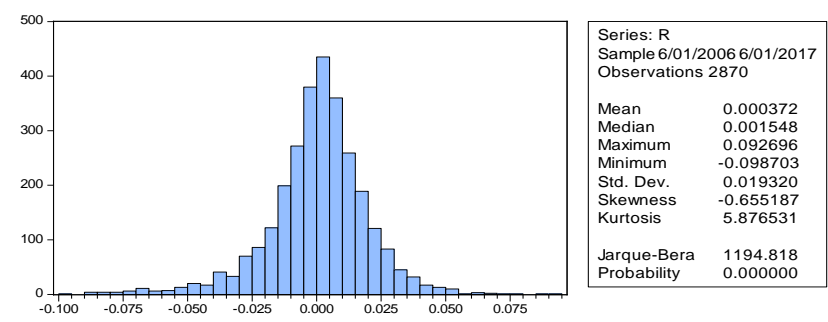

Fig. 2. Basic statistics of logarithmic return rate series of the SME Board index

By Fig. 2, in the sample range, The yield of the SME Board index has the following statistical characteristics: The mean value is positive 0.000372 , the median is 0.001548 , the maximum is 0.092696 , the minimum is -0.098703 , the standard deviation is 0.019320 , the skewness is less than 0 , the frequency of negative return is greater than positive rate, is a left trailing shape, the peak is 5.876531 is greater than the normal distribution of the standard value of 3 , indicating the characteristics of spikes, therefore, it can be preliminarily estimated that the yield of China's SME index has a sharp peak thick tail characteristics, do not obey the normal distribution. In addition, the JB statistic is 1194.818 far greater than the critical value and the $P$ value is 0.000000 , which indicates that the 
residual error should be rejected as a normal distribution, so the logarithmic return sequence does not obey the normal distribution.

\section{2) Test of smoothness}

\begin{tabular}{lccc}
\hline \hline & t-Statistic & \multirow{2}{*}{ Prob. $^{*}$} \\
\hline \hline Augmented Dickey-Fuller test statistic & -50.01604 & 0.0001 \\
\hline Test critical values: & $1 \%$ level & -3.432438 & \\
& $5 \%$ level & -2.862348 & \\
& $10 \%$ level & -2.567245 & \\
\hline \hline
\end{tabular}

Fig. 3. ADF Unit Root test

In this paper, the method of $\mathrm{ADF}$ unit root test is used to test the stability of the yield sequence of the SME. From Fig. 3, the check value of $t$ statistic of the logarithmic return sequence is less than the critical value of $1 \%, 5 \%$ and $10 \%$, and the $\mathrm{P}$ value tends to 0 , rejecting the original hypothesis that the unit root exists, so the logarithmic return sequence is a stationary sequence.

\section{3) Self-correlation test}

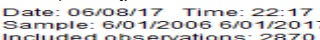

\begin{tabular}{|c|c|c|c|c|c|}
\hline Autacorrelation & Partial Correlation & AO & PAC & a-stat & Prob \\
\hline 娄 & 管 & 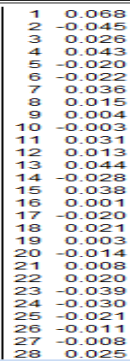 & 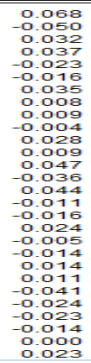 & 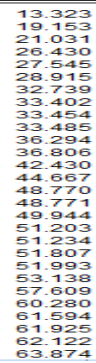 & 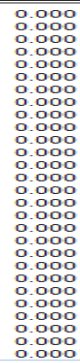 \\
\hline
\end{tabular}

Fig. 4. Self-correlation test of logarithmic return rate sequence of the SME Board index

As can be seen from fig. 4, the P-value corresponding to $\mathrm{Q}$ statistic tends to 0 , and the logarithmic yield sequence has autocorrelation.

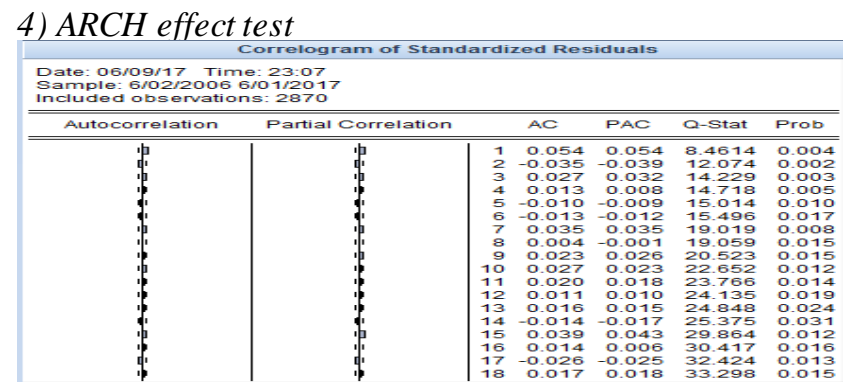

Fig. 5. Test of residual squared correlation graph for the logarithmic return rate of the SME Board index

In this paper, the method of residual square correlation graph is used to test the arch effect, and we can see from Fig. 5 that the logarithmic yield sequence has autocorrelation and arch effect.

\section{Establish a GARCH model}

\section{1) Order selection of $\operatorname{GARCH}(p, q)$}

According to the above statistical analysis result, this article establishes the GARCH (p,q) model, before modeling, should determine the $\mathrm{P}, \mathrm{Q}$ value first, its selection rule should follow the Red Pool information criterion (AIC) and the Schwartz criterion (SC).

TABLE I. GARCH(P, Q) ORDER TEST RESULTS

\begin{tabular}{|l|l|l|l|}
\hline & GARCH(1,1) & GARCH(1,2) & GARCH(2,1) \\
\hline AIC & -5.290772 & -5.290492 & -5.290808 \\
\hline SC & -5.284539 & -5.282183 & -5.282499 \\
\hline
\end{tabular}

From table 1, according to the $\mathrm{AIC}$ and $\mathrm{SC}$ minimum principle, this article chooses the GARCH $(1,1)$ modeling the effect to be better.

\section{2) ARCH-LM test of the obtained model}

TABLE II. ARCH-LM TEST RESUlTs

\begin{tabular}{|l|c|c|c|c|}
\hline & F-statistic & Prob.F & Obs.R-squared & Prob.Chi-Square \\
\hline GARCH(1,1)-N & 0.139696 & 0.7086 & 0.139787 & 0.7085 \\
\hline GARCH(1,1)-t & 0.028271 & 0.8665 & 0.02829 & 0.8664 \\
\hline GARCH(1,1)-GED & 0.002158 & 0.963 & 0.002159 & 0.9629 \\
\hline TARCH(1,1)-N & 0.131365 & 0.717 & 0.131451 & 0.7169 \\
\hline TARCH(1,1)-t & 0.035839 & 0.8499 & 0.035863 & 0.8498 \\
\hline TARCH(1,1)-GED & 0.000567 & 0.981 & 0.000568 & 0.981 \\
\hline EGARCH(1,1)-N & 0.333735 & 0.5635 & 0.333929 & 0.5634 \\
\hline EGARCH(1,1)-t & 0.024026 & 0.8768 & 0.024043 & 0.8768 \\
\hline EGARCH(1,1)-GED & 0.103601 & 0.7476 & 0.103669 & 0.7475 \\
\hline
\end{tabular}

It is shown from table 2 that the Garch family model with $\mathrm{T}$ distribution and GED distribution is close to 1 , and that the residual sequence of the model has no arch effect, which shows that the GARCH model has eliminated the variance of residual condition.

\section{3) Stability test of Garch model}

TABLE III. MODEL COEFFICIENTS AND Z-VALUES

\begin{tabular}{|c|c|c|c|c|}
\hline & $\boldsymbol{\alpha}$ & $\boldsymbol{\beta}$ & $\boldsymbol{\theta}$ & $\boldsymbol{\gamma}$ \\
\hline GARCH(1,1)-N & $2.88 \mathrm{E}-06$ & $\begin{array}{c}0.0509 \\
(11.7914)\end{array}$ & $\begin{array}{c}0.9405 \\
(217.1268)\end{array}$ & \\
\hline GARCH(1,1)-t & \multirow{2}{*}{$1.61 \mathrm{E}-06$} & $\begin{array}{c}0.0671 \\
(7.1033)\end{array}$ & $\begin{array}{c}0.9314 \\
(110.5633)\end{array}$ & \\
\hline \multirow{2}{*}{ GARCH(1,1)-GED } & $2.35 \mathrm{E}-06$ & $\begin{array}{c}0.0587 \\
(7.3940)\end{array}$ & $\begin{array}{c}0.9352 \\
(121.6127)\end{array}$ & \\
\hline \multirow{2}{*}{ TARCH(1,1)-N } & \multirow{2}{*}{$2.91 \mathrm{E}-06$} & $\begin{array}{c}0.0498 \\
(8.1697)\end{array}$ & $\begin{array}{c}0.0022 \\
(0.2928)\end{array}$ & $\begin{array}{c}0.9402 \\
(214.7943)\end{array}$ \\
\hline \multirow{2}{*}{ TARCH(1,1)-t } & \multirow{2}{*}{$1.68 \mathrm{E}-06$} & $\begin{array}{c}0.0617 \\
(4.8835)\end{array}$ & $\begin{array}{c}0.0104 \\
(0.7193)\end{array}$ & $\begin{array}{c}0.9310 \\
(109.3967)\end{array}$ \\
\hline TARCH(1,1)-GED & $2.42 \mathrm{E}-06$ & $\begin{array}{c}0.0552 \\
(5.0244)\end{array}$ & $\begin{array}{c}0.0071 \\
(0.5405)\end{array}$ & $\begin{array}{c}0.9347 \\
(119.6612)\end{array}$ \\
\hline \multirow{2}{*}{ EGARCH(1,1)-N } & \multirow{2}{*}{-0.1708} & $\begin{array}{c}0.1131(13.56 \\
90)\end{array}$ & $\begin{array}{c}-0.0156 \\
(-3.2867)\end{array}$ & $\begin{array}{c}0.9895 \\
(718.1269)\end{array}$ \\
\hline EGARCH(1,1)-t & -0.1572 & $\begin{array}{c}0.1388 \\
(8.3155)\end{array}$ & $\begin{array}{c}-0.0207 \\
(-2.2371)\end{array}$ & $\begin{array}{c}0.9936 \\
(365.0077)\end{array}$ \\
\hline EGARCH(1,1)-GED & -0.1658 & $\begin{array}{c}0.1262 \\
(8.5233)\end{array}$ & $\begin{array}{c}-0.0184 \\
(-2.2172)\end{array}$ & $\begin{array}{c}0.9914 \\
(398.3021)\end{array}$ \\
\hline
\end{tabular}


The ARCH and GARCH coefficients $\beta+\theta<1$ of the Garch $(1,1)$ model are shown in table 3, which shows that the variance equation satisfies the stability; the coefficient gamma of the lever effect term of the TARCH $(1,1)$ model is greater than 0 , indicating that the fluctuation of the yield has the lever effect; Egarch $(1,1)$ The non-symmetric gamma value of the model is more than 0 , which indicates that the positive news brings more fluctuation.

\section{Analysis of VaR value result}

TABLE IV. 99\% VAR VALUE OF DIFFERENT DIST RIBUTION MODELS UNDER CONFIDENCE DEGREE

\begin{tabular}{|c|l|l|l|l|l|l|}
\hline Distribution & GARCH class model & VaR mean & VaR median & VaR maximum & VaR minimum & VaR standard deviation \\
\hline \multirow{3}{*}{$\mathbf{N}$} & GARCH & 0.013128 & 0.100755 & 8.22054 & -6.212131 & 1.004378 \\
\cline { 2 - 7 } & TARCH & 0.013427 & 0.100037 & 8.173104 & -6.153273 & 1.004368 \\
\cline { 2 - 7 } & EGARCH & 0.012558 & 0.100124 & 8.096428 & -6.308843 & 1.00589 \\
\hline \multirow{3}{*}{$\mathbf{T}$} & GARCH & 0.015611 & 0.103602 & 7.469729 & -5.19981 & 0.998815 \\
\cline { 2 - 7 } & TARCH & 0.015674 & 0.103438 & 7.459798 & -5.191468 & 0.998844 \\
\cline { 2 - 7 } & EGARCH & 0.014303 & 0.099217 & 7.610191 & -5.625511 & 0.999099 \\
\hline \multirow{3}{*}{ GED } & GARCH & 0.014493 & 0.103106 & 7.83209 & -5.647986 & 1.004624 \\
\cline { 2 - 7 } & TARCH & 0.014701 & 0.102252 & 7.799284 & -5.614202 & 1.004685 \\
\cline { 2 - 7 } & EGARCH & 0.013461 & 0.100271 & 7.87908 & -5.988547 & 1.005599 \\
\hline
\end{tabular}

It can be seen from TABLE IV that under the three different hypotheses, the $\mathrm{T}$ distribution standard deviation is the smallest, which can better describe the characteristics of the yield, and the simulation results of the GARCH model are better under the same distribution.

\section{CONCLUSION}

As an important part of China's stock market, the paper takes the Garch family model to the normal test, the stationary test, the autocorrelation test and the arch test of the logarithmic return rate sequence of the SME, and then establishes the model, and makes the following conclusions:

The index yield of the SME board has the characteristics of sharp peak and thick tail, has the stability and the residual error has ARCH effect.

It is more accurate to GARCH model to fit the index yield sequence of China's SME, and can better eliminate the conditional variance.
At $99 \%$ confidence level, $\mathrm{T}$ distribution can better reflect the risk of China's SME market, and the GARCH $(1,1)$ model is more correct under the same distribution.

\section{REFERENCES}

[1] Autoregressive conditional heteroskedasticity with estimates of the variance of the United Kingdom inflation. Engle R F. Econometrica 1982 .

[2] Generalized autoregressive conditional heteroskedasticity. Bollerslev T. Journal of Econometrics. 1986

[3] Zhu Changyou, Hu Laifeng, Luoyang. Shanghai stock market and the dynamic relationship between the A and B-share market in Shenzhen -Based on the multiple DCC-GARCH model [J]. Journal of Chifeng University (Natural Science Edition), 2013, 29 (22): 86-88.

[4] Risk2: Measuring the risk in value at risk. Jorion, Philippe. Financial Analysts Journal. 1996

[5] GARCH 2.2: An Oxpackage for estimating and forecasting various ARCH models. LAURENT S PETERS J P. Journal of Econometrics. 2002

[6] A comparative study of VaR-GA RCH class models in stock market risk measurement $[\mathrm{J}]$. Huang Yanlong. Statistics \& Decision. 2008 (03) 